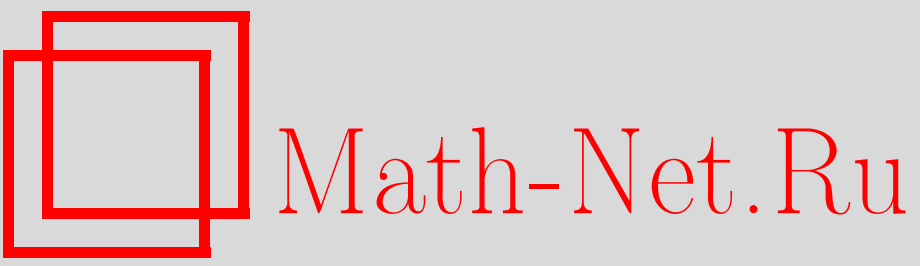

М. В. Куркина, Е. Д. Родионов, С. П. Семенов, В. В. Славский, Обобщенное преобразование Лежандра конформно плоских метрик, Итоги науки и техн. Сер. Соврем. мат. и ее прил. Темат. обз., 2020, том 182, 55-65

DOI: https://doi.org/10.36535/0233-6723-2020-182-55-65

Использование Общероссийского математического портала Math-Net.Ru подразумевает, что вы прочитали и согласны с пользовательским соглашением

http://www.mathnet.ru/rus/agreement

Параметры загрузки:

IP : 54.84 .234 .179

26 апреля 2023 г., 04:45:46 


\title{
ОБОБЩЕННОЕ ПРЕОБРАЗОВАНИЕ ЛЕЖАНДРА КОНФОРМНО ПЛОСКИХ МЕТРИК
}

\author{
(c) 2020 г. М. В. КУРКИНА, Е. Д. РОДИОНОВ, С. П. СЕМЕНОВ, В. В. СЛАВСКИЙ
}

\begin{abstract}
АннотАция. В вариационном исчислении важную роль играет двойственность Минковского, или преобразование Лежандра выпуклой функции. В работе рассмотрены слабо регулярные конформно плоские римановы метрики неотрицательной кривизны, определенные на $n$-мерной единичной сфере. Для данного класса метрик введен и подробно изучен аналог преобразования Лежандра.
\end{abstract}

Ключевъе слова: преобразование Лежандра, конформно плоская метрика, пространство Лобачевского.

\section{GENERALIZED LEGENDRE TRANSFORM OF CONFORMALLY FLAT METRICS}

\author{
(c) 2020 M. V. KURKINA, E. D. RODIONOV, S. P. SEMENOV, V. V. SLAVSKY
}

\begin{abstract}
In the calculus of variations, an important role is played by the Minkowski duality, or the Legendre transform of convex functions. We consider weakly regular, conformally flat Riemannian metrics of nonnegative curvature defined on the $n$-dimensional unit sphere. For this class of metrics, an analog of the Legendre transformation is introduced and studied in detail.
\end{abstract}

Keywords and phrases: Legendre transform, conformally flat metric, Lobachevsky space.

AMS Subject Classification: 53A07

1. Преобразование Лежандра регулярной конформно плоской метрики на сфере. Пусть $\mathbb{R}$ - числовая прямая, $\mathbb{R}^{n+1}$ - евклидово $(n+1)$-мерное арифметическое пространство, $M^{n+2}=\mathbb{R}^{n+1} \times \mathbb{R}$ - псевдоевклидово пространство, скалярный квадрат вектора $\boldsymbol{w}=[\boldsymbol{x}, \zeta] \in$ $M^{n+2}$ в котором равен $\langle\boldsymbol{w}\rangle^{2}=|\boldsymbol{x}|^{2}-\zeta^{2}$, где $|\boldsymbol{x}|^{2}$ - скалярный квадрат вектора $\boldsymbol{x} \in \mathbb{R}^{n+1}$. Обозначим через

$$
C^{+}=\left\{[\boldsymbol{x}, \zeta] \in M^{n+2}:|\boldsymbol{x}|^{2}-\zeta^{2}=0, \zeta>0\right\}
$$

верхнюю часть изотропного конуса в $M^{n+2}$. В дальнейшим, если будет ясно из контекста, мы будем обозначать $\boldsymbol{x}$ через $x$.

Лемма 1. Пусть на единичной сфере $S^{n} \subset \mathbb{R}^{n+1}$ задана конформно плоская метрика

$$
d s^{2}=\frac{d x^{2}}{f^{2}(x)}, \quad x \in S^{n} \subseteq \mathbb{R}^{n+1}
$$

Работа выполнена при поддержке Российского фонда фундаментальных исследований (проекты №o 18-47860016, 18-01-00620) и Научного Фонда Югорского государственного университета (проект № 13-01-20/10). 
где $f(x)$ - функиия класса $C^{1}$. Тогда определено каноническое изометрическое вложение, задаваемое формулой

$$
Z: x \in S^{n} \rightarrow\left[\frac{x}{f(x)}, \frac{1}{f(x)}\right] \in C^{+}
$$

Доказательство. Дифференцируя $Z$, получим:

$$
d Z=\left[\frac{d x}{f(x)}-x \frac{d f}{f^{2}(x)},-\frac{d f}{f^{2}(x)}\right] .
$$

Отсюда в силу равенства $(x, d x) \equiv 0$ имеем:

$$
\langle d Z, d Z\rangle=\frac{(d x, d x)}{f^{2}(x)}-2 \frac{(x, d x) d f}{f^{3}(x)}+\frac{(d f)^{2}}{f^{4}(x)}-\frac{(d f)^{2}}{f^{4}(x)}=\frac{(d x, d x)}{f^{2}(x)} .
$$

Замечание 1. Из доказательства видно, что регулярность конформно плоской метрики фактически не требуется.

Образ $Z\left(S^{n}\right)=F \subseteq C^{+}$- пространственноподобная $n$-мерная поверхность. В дальнейшем будем отождествлять конформно плоскую метрику с поверхностью $F$. Предположим, что функция $f(x)$ достаточно гладкая; тогда поверхность $F$ регулярна, и в каждой точке $Z(x) \in F$ определено $n$-мерное касательное пространство $T_{x}(F)$. Существует единственный вектор $Z^{*}(x) \in C^{+}$, для которого

$$
\left\langle Z, Z^{*}\right\rangle=-1, \quad Z^{*} \perp T_{x}(F),
$$

где ортогональность понимается относительно скалярного произведения в $M^{n+2}$.

Лемма 2. Пусть функиия $f(\boldsymbol{x})$, задающая конформно плоскую метрику, по однородности распространена на все пространство $\mathbb{R}^{n+1}$. Тогда вектор $Z^{*}$ явно выражается через $f$ u $\nabla f$ в $\mathbb{R}^{n+1}$ :

$$
Z^{*}(\boldsymbol{x})=\left[-\nabla f+\frac{|\nabla f|^{2}}{2 f} \boldsymbol{x}, \frac{|\nabla f|^{2}}{2 f}\right]
$$

где $\boldsymbol{x} \in S^{n} \subset \mathbb{R}^{n+1}, \nabla f$ - градиент функции $f$ в пространстве $\mathbb{R}^{n+1}$.

Доказательство. Из однородности $f(\boldsymbol{x})$ следует тождество Эйлера

$$
(\nabla f, \boldsymbol{x}) \equiv f(\boldsymbol{x})
$$

где $(\nabla f, \boldsymbol{x})$ - скалярное произведение в $\mathbb{R}^{n+1}$. Проверим $(2)$; имеем

$$
\begin{aligned}
\left\langle Z^{*}, Z^{*}\right\rangle & =\left(-\nabla f+\frac{|\nabla f|^{2}}{2 f} \boldsymbol{x}\right)^{2}-\left(\frac{|\nabla f|^{2}}{2 f}\right)^{2}=|\nabla f|^{2}-2(\nabla f, x) \frac{|\nabla f|^{2}}{2 f}+\frac{|\nabla f|^{4}}{4 f^{2}}-\frac{|\nabla f|^{4}}{4 f^{2}}=0, \\
\left\langle Z^{*}, Z\right\rangle & =\left(-\nabla f+\frac{|\nabla f|^{2}}{2 f} \boldsymbol{x}, \frac{x}{f(x)}\right)-\frac{|\nabla f|^{2}}{2 f^{2}}=-1, \\
\left\langle Z^{*}, d Z\right\rangle & =\left(-\nabla f+\frac{|\nabla f|^{2}}{2 f} \boldsymbol{x}, \frac{d x}{f(x)}-\frac{x d f}{f^{2}(x)}\right)+\frac{d f|\nabla f|^{2}}{2 f^{3}}=0 .
\end{aligned}
$$

Определение 1. Если точка $Z \in F$ пробегает поверхность $F$, то точка $Z^{*}$ пробегает двойственную поверхность $F^{*}$. Соответствующую конформно плоскую метрику

$$
d s^{* 2}=\frac{d y^{2}}{f^{* 2}(y)}, \quad y \in S^{n},
$$

будем называть полярной к исходной метрике (см. [6]). 
Сравнивая формулы (1) и (3), имеем:

$$
\left[-\nabla f+\frac{|\nabla f|^{2}}{2 f} \boldsymbol{x}, \frac{|\nabla f|^{2}}{2 f}\right] \equiv\left[\frac{y}{f^{*}(y)}, \frac{1}{f^{*}(y)}\right] .
$$

Отсюда получаем формулы для перехода к полярной конформно плоской метрике:

$$
f^{*}(y)=\frac{2 f(x)}{|\nabla f|^{2}}, \quad \boldsymbol{y}=\boldsymbol{x}-2 f(x) \frac{\nabla f}{|\nabla f|^{2}} .
$$

Лемма 3. Пусть $f: \mathbb{R}^{n+1} \rightarrow \mathbb{R}$ - произвольная однородная степени 1 функиия на $\mathbb{R}^{n+1}$. Отображение $H_{f}: S^{n} \rightarrow S^{n}$ определяемое формулой

$$
H_{f}: \boldsymbol{x} \in S^{n} \rightarrow \boldsymbol{x}-2 f(x) \frac{\nabla f}{|\nabla f|^{2}} \in S^{n}
$$

сохраняет норму вектора: $\left|H_{f}(\boldsymbol{x})\right|=|\boldsymbol{x}|$.

Доказательство. В силу однородности функции $f$ выполняется ортогональное разложение градиента

$$
\nabla f(x)=x f(x)+\nabla_{T} f(x), \quad x \perp \nabla_{T} f(x),
$$

где $\nabla_{T} f(x)$ - касательная составляющая градиента. Скалярный квадрат вектора $\boldsymbol{y}=H_{f}(\boldsymbol{x})$ равен

$$
(\boldsymbol{y}, \boldsymbol{y})=(\boldsymbol{x}, \boldsymbol{x})-4 f(x) \frac{(\nabla f, \boldsymbol{x})}{|\nabla f|^{2}}+4 f^{2}(x) \frac{(\nabla f, \nabla f)}{|\nabla f|^{4}}=(\boldsymbol{x}, \boldsymbol{x}) .
$$

Определение 2. Отображение $H_{f}$ назовем конформным градиентом функции $f$. Если отображение $H_{f}$ имеет обратное $H_{f}^{-1}$, то полярная метрика определяется функцией

$$
f^{*}(y)=\left.\frac{2 f(x)}{|\nabla f|^{2}}\right|_{x=H_{f}^{-1}(y)} .
$$

Замечание 2. Из определения 1 следует двойственность метрик

$$
d s^{2}=\frac{d x^{2}}{f^{2}(x)}, \quad x \in S^{n}, \quad \text { и } \quad d s^{* 2}=\frac{d y^{2}}{f^{* 2}(y)}, \quad y \in S^{n} .
$$

Поэтому при наличии соответствующей регулярности функции $f^{*}(y)$ будут справедливы равенства

$$
\begin{aligned}
f^{*}(y) & =\frac{2 f(x)}{|\nabla f(x)|^{2}}, & \boldsymbol{y} & =\boldsymbol{x}-2 f(x) \frac{\nabla f(x)}{|\nabla f(x)|^{2}}, \\
f(x) & =\frac{2 f^{*}(y)}{\left|\nabla f^{*}(y)\right|^{2}}, & \boldsymbol{x} & =\boldsymbol{y}-2 f^{*}(y) \frac{\nabla f^{*}(y)}{\left|\nabla f^{*}(y)\right|^{2}} .
\end{aligned}
$$

Следствие 1. Из (7) следуют тождества

$$
H_{f} \circ H_{f *}=I_{S^{n}}=H_{f *} \circ H_{f},
$$

т.е. преобразования сферы $H_{f}, H_{f *}$ являются взаимно обратными;

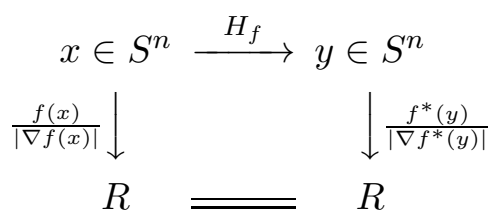

Доказательство. Действительно, складывая вторые равенства в (7), имеем

$$
2 f(x) \frac{\nabla f(x)}{|\nabla f(x)|^{2}}+2 f^{*}(y) \frac{\nabla f^{*}(y)}{\left|\nabla f^{*}(y)\right|^{2}} \equiv 0 ;
$$


следовательно, подставляя $H_{f}$ в $H_{f^{*}}$, получим первое тождество:

$$
x=\boldsymbol{y}-\left.2 f^{*}(y) \frac{\nabla f^{*}(y)}{\left|\nabla f^{*}(y)\right|^{2}}\right|_{\boldsymbol{y}=\boldsymbol{x}-2 f(x) \frac{\nabla f(x)}{|\nabla f(x)|^{2}}}=x .
$$

Взяв модули в (8), получим второе тождество.

\section{2. Преобразование Лежандра слабо регулярной конформно плоской метрики на cфepe.}

Теорема 1. Пусть $f(x)$-дифферениируемая функиия, $f^{*}(y)$ и у определень равенствами (4). Тогда справедливо тождество

$$
\|x-y\|^{2}=2 f(x) f^{*}(y)
$$

где $\|x-y\|$ - хордовое расстояние между точками единичной сферы $S^{n}$.

Доказательство. Из второго равенства (4) следует

$$
\|x-y\|^{2}=\frac{4 f^{2}(x)}{|\nabla f(x)|^{2}}=2 f(x) \frac{2 f(x)}{|\nabla f(x)|^{2}}=2 f(x) f^{*}(y) .
$$

Теорема 2. Пусть $f(x)$-дифферениируемая строго положительная функиия на единичной сфере, а отображение $H_{f}$ взаимно однозначно. Тогда для полярной функиии $f^{*}(y)$ справедливо равенство

$$
f^{*}(y)=\max _{x \in S^{n}} \frac{\|x-y\|^{2}}{2 f(x)}
$$

где $\|x-y\|$ - хордовое расстояние между точками единичной сферы $S^{n}$.

Доказательство. При фиксированном $y \in S^{n}$ функция $F(x)=\|x-y\|^{2} /(2 f(x))$ положительна и непрерывно дифференцируема по $x \in S^{n}$; следовательно, существует точка $x^{*} \in S^{n}$, в которой она принимает максимальное значение:

$$
\max _{x \in S^{n}} \frac{\|x-y\|^{2}}{2 f(x)}=\frac{\left\|x^{*}-y\right\|^{2}}{2 f\left(x^{*}\right)} .
$$

Дифференциал функции $F(x)$ равен

$$
d F=\frac{(x-y, d x)}{f(x)}-\frac{d f\|x-y\|^{2}}{2 f(x)^{2}} .
$$

Следовательно, в точке $x^{*} \in S^{n}$ выполняется равенство

$$
\frac{x^{*}-y}{f\left(x^{*}\right)}=\frac{\nabla f\left(x^{*}\right)\left\|x^{*}-y\right\|^{2}}{2 f\left(x^{*}\right)^{2}}
$$

или

$$
\frac{x^{*}-y}{\left\|x^{*}-y\right\|^{2}}=\frac{\nabla f\left(x^{*}\right)}{2 f\left(x^{*}\right)} .
$$

Применяя инверсию относительно единичной сферы с центром в начале координат к левой и правой части (10), получим:

$$
x^{*}-y=2 f\left(x^{*}\right) \frac{\nabla f\left(x^{*}\right)}{\left\|\nabla f\left(x^{*}\right)\right\|^{2}} \quad \text { или } \quad y=x^{*}-2 f\left(x^{*}\right) \frac{\nabla f\left(x^{*}\right)}{\left\|\nabla f\left(x^{*}\right)\right\|^{2}}=H_{f}\left(x^{*}\right) ;
$$

следовательно, $x^{*}=H_{f}^{-1}(y)$. Из равенства (10) получаем

$$
F\left(x^{*}\right)=\frac{\left\|x^{*}-y\right\|^{2}}{2 f\left(x^{*}\right)}=\left.\frac{2 f\left(x^{*}\right)}{\left\|\nabla f\left(x^{*}\right)\right\|^{2}}\right|_{x^{*}=H_{f}^{-1}(y)}=f^{*}(y) .
$$


Следствие 2. В условиях теоремы функиии $f(x), f^{*}(y)$ положительны и выполняется неравенство

$$
\|x-y\|^{2} \leqslant 2 f(x) f^{*}(y)
$$

где $x, y \in S^{n}$ - произвольные точки единичной сферы, $\|x-y\|-$-хордовое расстояние. Неравенство (11) можно рассматривать как аналог неравенства Юнга-Фенхеля в выпуклом анализе.

Замечание 3. В условиях теоремы функция $f^{*}(y)$ также будет дифференцируема и строго положительна, а отображение $H_{f^{*}}=H_{f}^{-1}$ взаимно однозначно; поэтому

$$
f(x)=\max _{y \in S^{n}} \frac{\|x-y\|^{2}}{2 f^{*}(y)} .
$$

Формулы (9)-(12) позволяют определить преобразование Лежандра для слабо регулярных конформно плоских метрик.

Теорема 3. В условиях теоремы функиии $g(x)=\sqrt{f(x)} u g^{*}(y)=\sqrt{f^{*}(y)}$ удовлетворяют трехточечным неравенствам

$$
\begin{gathered}
g(x) \leqslant g\left(x_{1}\right) \frac{\left|x_{2}-x\right|}{\left|x_{2}-x_{1}\right|}+g\left(x_{2}\right) \frac{\left|x-x_{1}\right|}{\left|x_{2}-x_{1}\right|}, \\
g^{*}(y) \leqslant g^{*}\left(y_{1}\right) \frac{\left|y_{2}-y\right|}{\left|y_{2}-y_{1}\right|}+g^{*}\left(y_{2}\right) \frac{\left|y-y_{1}\right|}{\left|y_{2}-y_{1}\right|},
\end{gathered}
$$

для любой тройки точек $x_{1}, x, x_{2} \in S^{n}$.

Доказательство. В силу неравенства Птолемея для любой четверки точек $x, y, y_{1}, y_{2} \in S^{n}$ выполняется неравенство

$$
|x-y| \leqslant \frac{\left|x-y_{1}\right|\left|y_{2}-y\right|}{\left|y_{2}-y_{1}\right|}+\frac{\left|x-y_{2}\right|\left|y-y_{1}\right|}{\left|y_{2}-y_{1}\right|} .
$$

Разделим это неравенство на $\sqrt{2} g(x)$ :

$$
\frac{|x-y|}{\sqrt{2} g(x)} \leqslant \frac{\left|x-y_{1}\right|\left|y_{2}-y\right|}{\sqrt{2} g(x)\left|y_{2}-y_{1}\right|}+\frac{\left|x-y_{2}\right|\left|y-y_{1}\right|}{\sqrt{2} g(x)\left|y_{2}-y_{1}\right|},
$$

и положим $x=H_{f^{*}}(y)$; тогда из теоремы (1) и неравенства (11) следует

$$
\frac{|x-y|}{\sqrt{2} g(x)}=g^{*}(y), \quad \frac{\left|x-y_{1}\right|}{\sqrt{2} g(x)} \leqslant g^{*}\left(y_{1}\right), \quad \frac{\left|x-y_{2}\right|}{\sqrt{2} g(x)} \leqslant g^{*}\left(y_{2}\right) .
$$

Отсюда получим

$$
g^{*}(y) \leqslant g^{*}\left(y_{1}\right) \frac{\left|y_{2}-y\right|}{\left|y_{2}-y_{1}\right|}+g^{*}\left(y_{2}\right) \frac{\left|y-y_{1}\right|}{\left|y_{2}-y_{1}\right|} .
$$

Аналогично доказывается первое неравенство в (13).

Следствие 3. В условиях теоремы функиии $g(x), g^{*}(y)$ будут конформно выпуклыми, и одномерная секиионная кривизна соответствующих конформно плоских метрик положительна (см. [3]); верно также обратное утверждение.

3. Преобразование Лежандра слабо регулярной конформно плоской метрики, определенной на $\mathbb{R}^{n}$. Задание конформно плоской метрики на сфере в смысле приложений не совсем удобно, и ее часто задают в евклидовом пространстве (см. [4]). Поэтому рассмотрим евклидов случай еще раз отдельно. В плоском случае метрика определяется выражением вида $d s^{2}=d x^{2} / f^{2}(x)$, где $f \in C^{1}\left(\mathbb{R}^{n}\right)$ - положительная функция.

Определение 3. Одномерная секционная кривизна конформно плоской метрики $d s^{2}=$ $d x^{2} / f^{2}(x)$ в $\mathbb{R}^{n}$ задается формулой

$$
K_{1 / 2}(f, x, \xi)=f \frac{d^{2} f}{d \xi^{2}}-\frac{1}{2}|\nabla f|^{2}
$$


(см. $[1,5,9]$ ); здесь $d^{2} f / d \xi^{2}$ - вторая производная функции в точке $x \in \mathbb{R}^{n}$ вдоль единичного вектора $\xi, \nabla f$-градиент функции $f$ в $\mathbb{R}^{n}$. Формула верна как в плоском случае, так и для единичной сферы; в последнем случае функция $f: S^{n} \rightarrow \mathbb{R}$ продолжается по однородности на $\mathbb{R}^{n+1}, x \in S^{n} \subset \mathbb{R}^{n+1}, \xi$ - единичный касательный вектор к сфере в точке $x, \nabla f$-градиент функции в $\mathbb{R}^{n+1}$.

Замечание 4. Конформно плоская метрика имеет постоянную одномерную кривизну если и только если функция $f(x)$ (для плоской модели) имеет вид квадратичного полинома

$$
f(x)=a\|x\|^{2}+\sqrt{2}(x, \boldsymbol{b})+c ;
$$

при этом одномерная кривизна вычисляется по формуле

$$
K_{1 / 2} \equiv \frac{-\langle w, w\rangle}{2}
$$

где $\langle w, w\rangle=\|\boldsymbol{b}\|^{2}-2 a c-$ скалярный квадрат вектора $w=[\boldsymbol{b}, a, c] \in M^{n+2}$ в псевдоевклидовом пространстве $M^{n+2}=\mathbb{R}^{n} \times \mathbb{R} \times \mathbb{R}$, снабженном скалярным произведением

$$
\left\langle w_{1}, w_{2}\right\rangle=\left(\boldsymbol{b}_{1}, \boldsymbol{b}_{2}\right)-a_{1} c_{2}-a_{2} c_{1} .
$$

Пример 1. В сферическом случае одномерная секционная кривизна метрики $d s^{2}=d x^{2} / f^{2}(x)$, где $f(x)=\varepsilon|x|+(b, x), x \in S^{n} \subset \mathbb{R}^{n+1}$, равна

$$
\begin{aligned}
K_{1 / 2}(f, x, \xi)= & f \frac{d^{2} f}{d \xi^{2}}-\frac{1}{2}|\nabla f|^{2}= \\
& =(\varepsilon|x|+(b, x)) \varepsilon\left[\frac{|\xi|^{2}}{|x|}-\frac{(x, \xi)^{2}}{|x|^{3}}\right]-\frac{1}{2}\left[\frac{\varepsilon^{2}(x, x)}{|x|^{2}}+|b|^{2}+\frac{2 \varepsilon(b, x)}{|x|}\right]=\frac{1}{2}\left[\varepsilon^{2}-|b|^{2}\right],
\end{aligned}
$$

где $|\xi|=1, \xi \perp x,|x|=1$.

Определение 4. Пусть $\kappa>0$. Определим поверхности

$$
\begin{aligned}
& C^{+}=\left\{w=[\boldsymbol{b}, a, c] \in M^{n+2}:\langle w, w\rangle=0, a>0 \vee c>0\right\}, \\
& H_{\kappa}=\left\{w=[\boldsymbol{b}, a, c] \in M^{n+2}:\langle w, w\rangle=-1 / \kappa, a>0 \vee c>0\right\},
\end{aligned}
$$

где $C^{+} \subset M^{n+2}$ - положительная пола изотропного конуса, поверхность $H_{\kappa}$ - положительная пола двуполостного гиперболоида (вместе с индуцированной метрикой из $M^{n+2}$ это пространство Лобачевского кривизны $(-\kappa))$.

Произвольной конформно плоской метрике $d s^{2}=d x^{2} / f^{2}(x)$ поставим в соответствие вложение

$$
Z(x)=\frac{1}{f(x)}\left[-x, \frac{1}{\sqrt{2}}, \frac{\|x\|^{2}}{\sqrt{2}}\right] \in C^{+},
$$

и двойственное, или сопряженное отображение

$$
Z^{*}(x)=\left[\frac{2 f \nabla f-x\|\nabla f\|^{2}}{2 f}, \frac{\|\nabla f\|^{2}}{2 \sqrt{2} f}, \frac{\left(2 f \nabla f-x\|\nabla f\|^{2}\right)^{2}}{2 \sqrt{2} f\|\nabla f\|^{2}}\right] \in C^{+} .
$$

Справедливы равенства

$$
\begin{gathered}
\left\langle Z(x), Z^{*}(x)\right\rangle=-1, \quad\left\langle d Z(x), Z^{*}(x)\right\rangle=0 \\
\langle d Z(x), d Z(x)\rangle=\frac{(d x, d x)}{f(x)^{2}}, \quad\left\langle d Z^{*}(x), d Z^{*}(x)\right\rangle=\frac{(A d x, A d x)}{f(x)^{2}},
\end{gathered}
$$

где симметричная матрица $A$ определяется квадратичной формой

$$
(A \xi, \xi)=f(x) d^{2} f(\xi, \xi)-\frac{1}{2}|\nabla f|^{2}|\xi|^{2} .
$$

Как и для сферической модели отображение $Z$ изометрично вкладывает метрику $d s^{2}=d x^{2} / f^{2}(x)$, $x \in \mathbb{R}^{n}$, в изотропный конус $C^{+}$, а отображение $Z^{*}$ изометрично вкладывает двойственную метрику $d s^{* 2}=d y^{2} / f^{* 2}(y), y \in \mathbb{R}^{n}$, в изотропный конус $C^{+}$. 
Сравнивая формулы (16) и (17), получаем равенство

$$
\left[\frac{2 f \nabla f-x\|\nabla f\|^{2}}{2 f}, \frac{\|\nabla f\|^{2}}{2 \sqrt{2} f}, \frac{\left(2 f \nabla f-x\|\nabla f\|^{2}\right)^{2}}{2 \sqrt{2} f\|\nabla f\|^{2}}\right] \equiv \frac{1}{f^{*}(y)}\left[-y, \frac{1}{\sqrt{2}}, \frac{\|y\|^{2}}{\sqrt{2}}\right],
$$

откуда находим формулы для перехода к полярной метрике в плоской модели:

$$
f^{*}(y)=\frac{2 f(x)}{|\nabla f|^{2}}, \quad \boldsymbol{y}=\boldsymbol{x}-2 f(x) \frac{\nabla f}{|\nabla f|^{2}} .
$$

Пусть $f: \mathbb{R}^{n} \rightarrow \mathbb{R}$ - произвольная функция. Отображение $H_{f}: \mathbb{R}^{n} \rightarrow \mathbb{R}^{n}$, определяемое формулой

$$
H_{f}: \boldsymbol{x} \rightarrow \boldsymbol{x}-2 f(x) \frac{\nabla f}{|\nabla f|^{2}},
$$

как и для сферической модели, будем называть конформным градиентом функции $f$.

Формулы (19), (5) формально совпадают, но здесь $x, y \in \mathbb{R}^{n}$, функция $f$ произвольная (не обязательно однородная степени один). Также сохраняют свой смысл теорема 1 и формула (9).

Пример 2. Для двумерного случая $d s^{2}=d x^{2} / f\left(x_{1}, x_{2}\right)^{2}$ формулы примут вид

$$
H_{f}=\left\{\begin{array}{l}
y_{1}=x_{1}-\frac{2 f\left(x_{1}, x_{2}\right) f^{(1,0)}\left(x_{1}, x_{2}\right)}{f^{(0,1)}\left(x_{1}, x_{2}\right)^{2}+f^{(1,0)}\left(x_{1}, x_{2}\right)^{2}} \\
y_{2}=x_{2}-\frac{2 f\left(x_{1}, x_{2}\right) f^{(0,1)}\left(x_{1}, x_{2}\right)}{f^{(0,1)}\left(x_{1}, x_{2}\right)^{2}+f^{(1,0)}\left(x_{1}, x_{2}\right)^{2}}
\end{array}\right.
$$

В силу двойственности полярного преобразования аналогичные соотношения выполняются для полярной метрики $d s^{* 2}=d y^{2} / f^{*}\left(y_{1}, y_{2}\right)^{2}$ :

$$
H_{f^{*}}=\left\{\begin{array}{l}
x_{1}=y_{1}-\frac{2 f^{*}\left(y_{1}, y_{2}\right) f^{*(1,0)}\left(y_{1}, y_{2}\right)}{f^{*(0,1)}\left(y_{1}, y_{2}\right)^{2}+f^{*(1,0)}\left(y_{1}, y_{2}\right)^{2}} \\
x_{2}=y_{2}-\frac{2 f^{*}\left(y_{1}, y_{2}\right) f^{*(0,1)}\left(y_{1}, y_{2}\right)}{f^{*(0,1)}\left(y_{1}, y_{2}\right)^{2}+f^{*(1,0)}\left(y_{1}, y_{2}\right)^{2}} .
\end{array}\right.
$$

3.1. Преобразование Лежандра одномерной конформно плоской метрики. Формально можно рассматривать также одномерные конформно плоские метрики, т.е. метрики вида

$$
d s^{2}=\frac{d x^{2}}{f^{2}(x)}, \quad x \in \mathbb{R}^{1}, \quad \text { или } \quad d s^{2}=\frac{d \varphi^{2}}{f^{2}(\varphi)}, \quad \varphi \in S^{1},
$$

с одномерной секционной кривизной:

$$
K_{1 / 2}=f \cdot \frac{d^{2} f}{d x^{2}}-\frac{1}{2}\left(\frac{d f}{d x}\right)^{2} \quad \text { или } \quad K_{1 / 2}=f \cdot \frac{d^{2} f}{d \varphi^{2}}-\frac{1}{2}\left(\frac{d f}{d \varphi}\right)^{2}+\frac{1}{2} f^{2} .
$$

Можно рассматривать их просто как функции, заданные на числовой прямой или на окружности, либо интерпретировать как сигнал (периодический или нет во времени). При $n=2$ возможна также интерпретация как изображения.

Для одномерной конформно плоской метрики $d s^{2}=d x^{2} / f^{2}(x)$, т.е. метрики на прямой $x \in \mathbb{R}$, формулы (16), (17) упрощаются:

$$
\begin{gathered}
Z(x)=\frac{1}{f(x)}\left[-x, \frac{1}{\sqrt{2}}, \frac{x^{2}}{\sqrt{2}}\right] \in C^{+}, \\
Z^{*}(x)=\left[\frac{2 f \cdot f^{\prime}-x \cdot f^{\prime 2}}{2 f}, \frac{f^{\prime 2}}{2 \sqrt{2} f}, \frac{\left(2 f \cdot f^{\prime}-x \cdot f^{\prime 2}\right)^{2}}{2 \sqrt{2} f \cdot f^{\prime 2}}\right] \in C^{+} .
\end{gathered}
$$

Соответственно формулы (18) перехода к полярной метрике примут вид

$$
f^{*}(y)=\frac{2 f(x)}{f^{\prime}(x)^{2}}, \quad y=x-\frac{2 f(x)}{f^{\prime}(x)},
$$




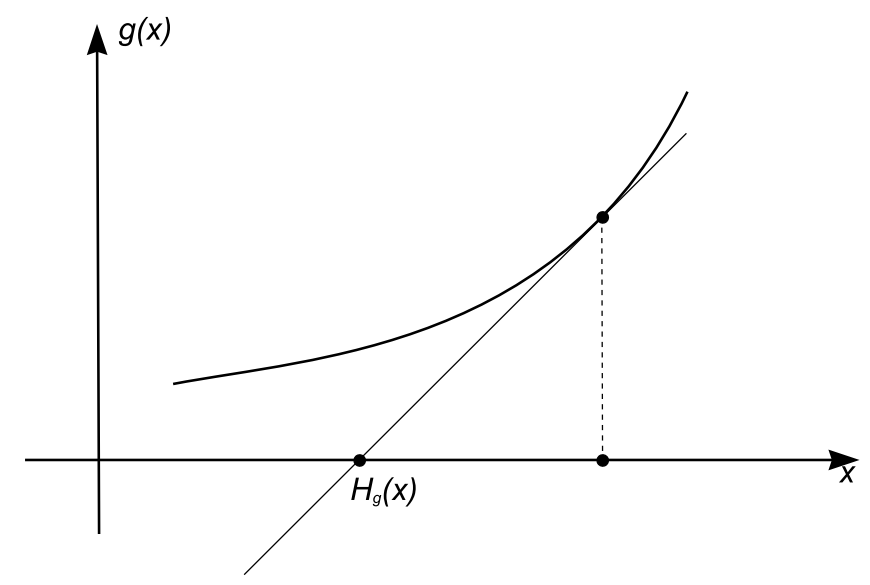

Рис. 1 . K замечанию 5: построение отображения $y=H_{g}(x)$ по графику функции $g(x)=\sqrt{f(x)}$

а отображение (19) -

$$
H_{f}: x \rightarrow y=x-\frac{2 f(x)}{f^{\prime}(x)} .
$$

Замечание 5. Преобразованию (18) можно придать ясный геометрический смысл (см. рис. 1), если перейти от функции $f(x)$ к функции $g(x)=\sqrt{f(x)}, f(x)=g^{2}(x)$. Тогда формулы преобразования примут вид

$$
g^{*}(y)=\frac{1}{\sqrt{2}\|\nabla g(x)\|}, \quad y=H_{g}(x)=x-\frac{g(x) \nabla g(x)}{\|\nabla g(x)\|^{2}} .
$$

В терминах функции $g(x)=\sqrt{f(x)}$ формулы принимают вид

$$
g^{*}(y)=\frac{1}{\sqrt{2}\left|g^{\prime}(x)\right|}, \quad y=H_{g}(x)=x-\frac{g(x)}{g^{\prime}(x)},
$$

а формулы (11), (9) - вид

$$
|x-y| \leqslant \sqrt{2} g(x) g^{*}(y), \quad g^{*}(y)=\max _{x} \frac{|x-y|}{\sqrt{2} g(x)}, \quad g(x)=\max _{y} \frac{|x-y|}{\sqrt{2} g^{*}(y)} .
$$

Пример 3. Пусть

$$
g(x)=\frac{1}{5}|x-2|+\frac{3}{10}|x+1|+\frac{2}{5}|x+2| ;
$$

график функции $g(x)$ изображен на рис. 2(а). Полярное преобразование $g^{*}(y)$ имеет вид:

$$
\begin{aligned}
y & =x-\frac{\frac{1}{5}|x-2|+\frac{3}{10}|x+1|+\frac{2}{5}|x+2|}{\frac{1}{5} \operatorname{sign}(x-2)+\frac{3}{10} \operatorname{sign}(x+1)+\frac{2}{5} \operatorname{sign}(x+2)}, \\
g^{*}(y) & =\frac{1}{\sqrt{2}\left|\frac{1}{5} \operatorname{sign}(x-2)+\frac{3}{10} \operatorname{sign}(x+1)+\frac{2}{5} \operatorname{sign}(x+2)\right|} ;
\end{aligned}
$$

последние два равенства определяют двойственную функцию $g^{*}(y)$ в параметрическом виде. В явном виде

$$
g^{*}(y)=\frac{|y-9|}{22 \sqrt{2}}+\frac{18 \sqrt{2}\left|y+\frac{7}{9}\right|}{55}+\frac{3|y+3|}{10 \sqrt{2}}
$$

график представлен на рисунке 2(b). 


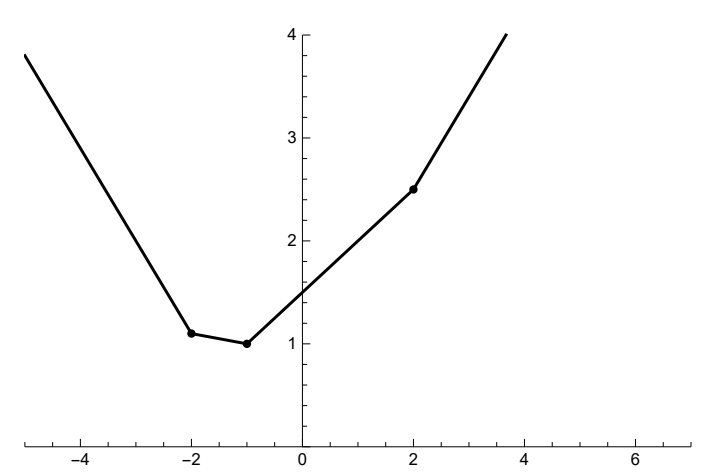

(a)

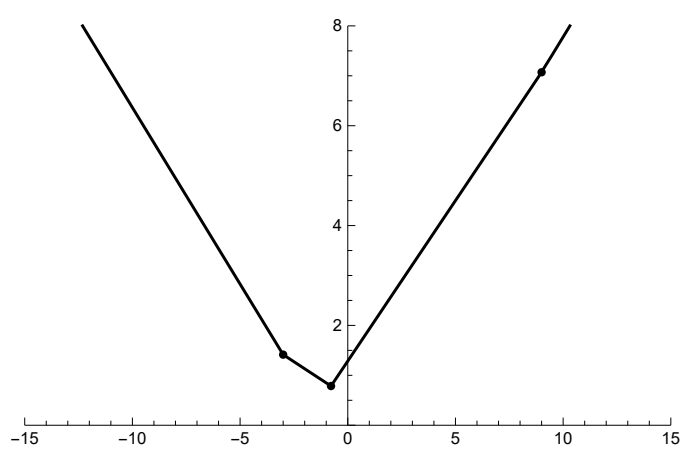

(b)

Рис. 2. K примеру 3: графики функций $g(x)$ (слева) и $g^{*}(y)$ (справа)

Формулы (21) соответственно принимают вид:

$$
\begin{gathered}
\frac{|y-9|}{22 \sqrt{2}}+\frac{18 \sqrt{2}\left|y+\frac{7}{9}\right|}{55}+\frac{3|y+3|}{10 \sqrt{2}} \equiv \max _{x} \frac{|y-x|}{\sqrt{2}\left(\frac{1}{5}|x-2|+\frac{3}{10}|x+1|+\frac{2}{5}|x+2|\right)}, \\
\frac{1}{5}|x-2|+\frac{3}{10}|x+1|+\frac{2}{5}|x+2| \equiv \max _{y} \frac{|y-x|}{\sqrt{2}\left(\frac{|y-9|}{22 \sqrt{2}}+\frac{18 \sqrt{2}\left|y+\frac{7}{9}\right|}{55}+\frac{3|y+3|}{10 \sqrt{2}}\right)}, \\
|y-x| \leqslant \sqrt{2}\left(\frac{|y-9|}{22 \sqrt{2}}+\frac{18 \sqrt{2}\left|y+\frac{7}{9}\right|}{55}+\frac{3|y+3|}{10 \sqrt{2}}\right) \times\left(\frac{1}{5}|x-2|+\frac{3}{10}|x+1|+\frac{2}{5}|x+2|\right) .
\end{gathered}
$$

Замечание 6. Пусть $\left\{\left(a_{i}, \alpha_{i}\right): i=1, \ldots, p\right\}$ - конечный набор точек числовой прямой с положительными весами $\left\{\alpha_{i}>0: i=1, \ldots, p\right\}$; тогда функция

$$
g(x)=\sum_{i=1, \ldots, p} \alpha_{i}\left|x-a_{i}\right|
$$

конформно выпукла. Двойственная к ней функция имеет аналогичный вид:

$$
g^{*}(y)=\sum_{j=1, \ldots, s} \beta_{j}\left|y-b_{j}\right|
$$

причем выполняется тождество

$$
\sum_{j=1, \ldots, s} \beta_{j}\left|y-b_{j}\right| \equiv \max _{x} \frac{|y-x|}{\sqrt{2} \sum_{i=1, \ldots, p} \alpha_{i}\left|x-a_{i}\right|} .
$$

3.2. Численная реализачия преобразования Лежандра конформно выпуклых функиий в системе MatLab. Пусть $d s^{2}=d \varphi^{2} / h^{2}(\varphi)$ - одномерная конформно плоская метрика, заданная на единичной окружности $0 \leqslant \varphi \leqslant 2 \pi$, где $h(\varphi)$ - гладкая периодическая функция. Обозначим через $f(x)$, $x \in \mathbb{R}^{2}$, однородное степени 1 продолжение функции $h$ на плоскость $\mathbb{R}^{2}$. Тогда на единичной окружности

$$
\begin{gathered}
x=[\cos (\varphi), \sin (\varphi)], \quad f(x)=h(\varphi) \\
\nabla f=h(\varphi)[\cos (\varphi), \sin (\varphi)]+h^{\prime}(\varphi)[-\sin (\varphi), \cos (\varphi)], \quad|\nabla f|^{2}=h(\varphi)^{2}+h^{\prime}(\varphi)^{2} .
\end{gathered}
$$


Положив $y=\left[\cos \left(\varphi^{*}\right), \sin \left(\varphi^{*}\right)\right]$, получим формулы преобразования Лежандра для функции $h$ на окружности:

$$
\begin{aligned}
\cos \left(\varphi^{*}\right) & =\frac{\left[h^{\prime 2}(\varphi)-h^{2}(\varphi)\right] \cos (\varphi)+2 h(\varphi) h^{\prime}(\varphi) \sin (\varphi)}{h^{2}(\varphi)+h^{\prime 2}(\varphi)} \\
\sin \left(\varphi^{*}\right) & =\frac{-2 h(\varphi) h^{\prime}(\varphi) \cos (\varphi)+\left[h^{\prime 2}(\varphi)-h^{2}(\varphi)\right] \sin (\varphi)}{h^{2}(\varphi)+h^{\prime 2}(\varphi)} \\
h^{*}\left(\varphi^{*}\right) & =\frac{2 h(\varphi)}{h(\varphi)^{2}+h^{\prime}(\varphi)^{2}} .
\end{aligned}
$$

Равенства (9) примут вид

$$
\begin{aligned}
h^{*}\left(\varphi^{*}\right) & =\max _{\varphi \in S^{1}} \frac{2 \sin ^{2}\left(\left(\varphi-\varphi^{*}\right) / 2\right)}{h(\varphi)}, \\
h(\varphi) & =\max _{\varphi^{*} \in S^{1}} \frac{2 \sin ^{2}\left(\left(\varphi^{*}-\varphi\right) / 2\right)}{h^{*}\left(\varphi^{*}\right)} .
\end{aligned}
$$

Ниже приведена m-функция в системе MatLab, реализующая формулу (26) преобразования Лежандра на окружности:

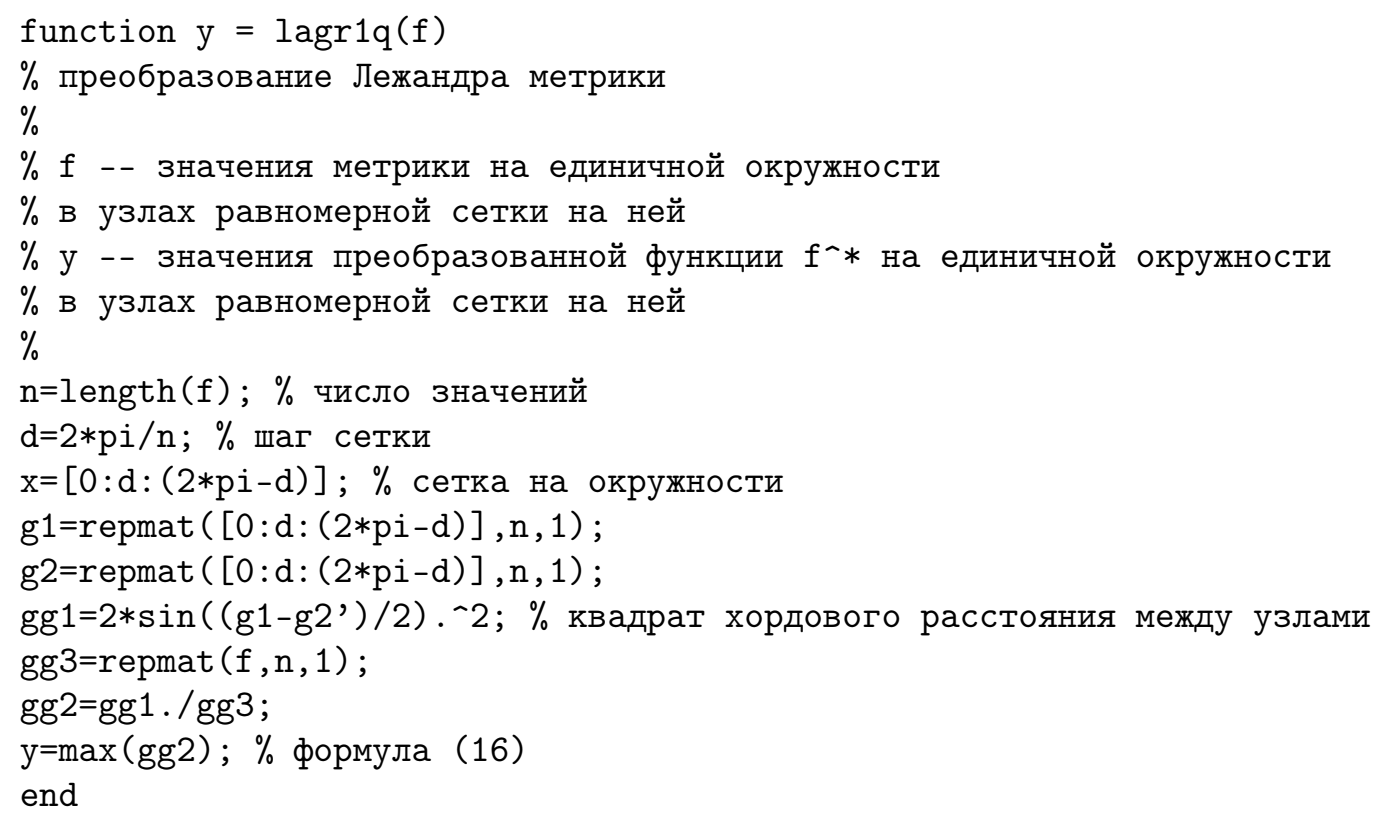

\section{СПИСОК ЛИТЕРАТУРЫ}

1. Балащенко В. В., Никоноров Ю. Г., Родионов Е. Д., Славский В. В. Однородные пространства: теория и приложения. - Ханты-Мансийск: Полиграфист, 2008.

2. Куркина М. В., Семенов С. П., Славский В. В. Численная реализация преобразования Лежандра конформно выпуклых функций// Сб. науч. тр. междунар. конф. «Ломоносовские чтения на Алтае. Фундаментальные проблемы науки и техники» (Барнаул, 13-16 ноября 2018 г.). - Барнаул: Алтайский государственный университет, 2018. - С. 321-326.

3. Куркина М. В., Родионов Е. Д., Славский В. В. Конформно выпуклые функции и конформно плоские метрики неотрицательной кривизны// Докл. РАН. - 2015. - 462, № 2. - С. 141--143.

4. Решетняк Ю. Г. Теоремы устойчивости в геометрии и анализе. - Новосибирск: Изд-во Ин-та мат. CO PAH, 1996.

5. Родионов Е. Д., Славский В. В. Одномерная секционная кривизна римановых многообразий// Докл. PAH. -2002 . -387 , № 4. - C. 454-457.

6. Славский В. В. Конформно плоские метрики ограниченной кривизны на $n$-мерной сфере// в кн.: Исследования по геометрии «в целом» и математическому анализу. - Новосибирск: Наука, 1987. C. $183-199$. 
7. Славский В. В. Конформно-плоские метрики и псевдоевклидова геометрия// Сиб. мат. ж. - 1994. 35, № 3. - С. 674-682.

8. Топоногов B. A. Дифференциальная геометрия кривых и поверхностей. - М.: Физматкнига, 2012.

9. Nikonorov Yu. G., Rodionov E. D., Slavskii V. V. Geometry of homogeneoues Riemannian manifolds// J. Math. Sci. - 2007. - 146, № 6. - P. 6313-6390.

Куркина Мария Викторовна

Югорский государственный университет, Ханты-Мансийск

E-mail: mavi@inbox.ru

Родионов Евгений Дмитриевич

Алтайский государственный университет, Барнаул

E-mail: edr2002@mail.ru

Семенов Сергей Петрович

Югорский государственный университет, Ханты-Мансийск

E-mail: ssp@ugrasu.ru

Славский Виктор Владимирович

Югорский государственный университет, Ханты-Мансийск

E-mail: slavsky2004@mail.ru 